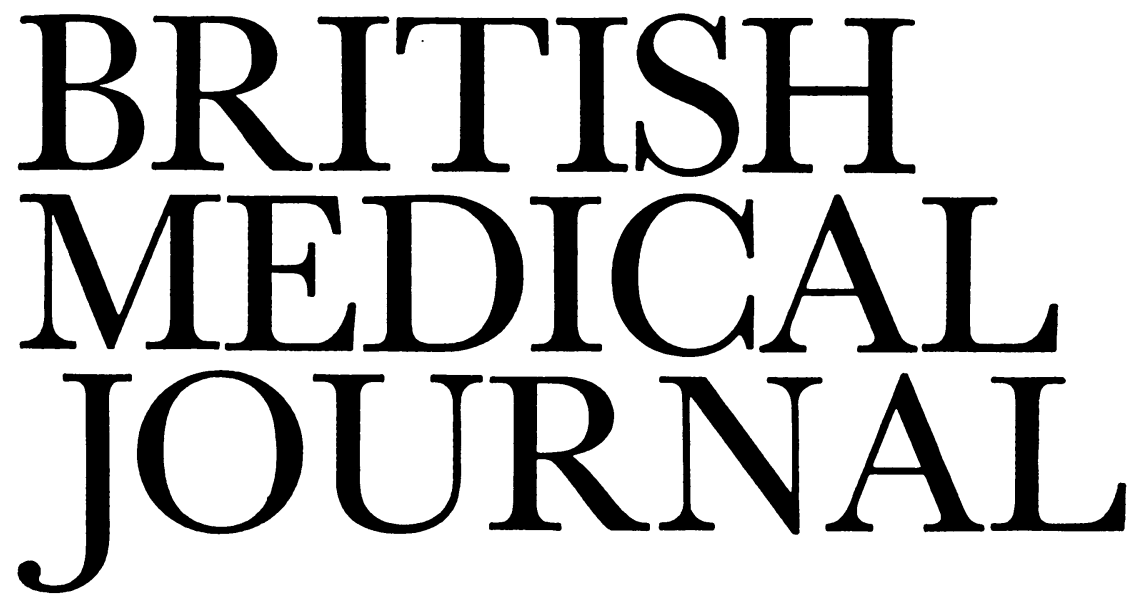

LONDON SATURDAY 3 APRIL 1971

\title{
Pathogenesis of Myasthenia Gravis
}

Myasthenia gravis is now considered to be an autoimmune disorder in the same sense as Graves's disease or the sialoadenitis of Sjogren's syndrome. The cause of these diseases is unknown, but the lesions can be explained on the hypothesis that patients have an abnormal immune response against their own tissues. Thus it is possible to speak of an autoimmune pathogenesis without knowing the initiating agent or other possible causes.

The striking resemblance of the lymphoid hyperplasia found in the thymic medulla of patients with myasthenia gravis to that seen in the thyroid in Hashimoto's disease first suggested an autoimmune link between the two disorders. ${ }^{1}$ Clinical relationships with other autoimmune diseases also favoured this hypothesis. ${ }^{2}$ It obtained further support when antibodies to muscle were detected in the serum of myasthenic patients $^{3}$ and antigenic cross-reaction was found between them and myoid cells in the thymus. ${ }^{4}$ The beneficial effect of thymectomy was already evident, ${ }^{5}$ and the occurrence of transient neonatal myasthenia in children of mothers with the disease had suggested that an inhibitor of neuromuscular transmission was circulating in the blood. ${ }^{6}$

However, none of the detectable antibodies react with the neural end-plate, and soluble complexes such as those obstructing the renal glomeruli in nephritis could never be found at the neuromuscular junction. It was only when refined neurophysiological techniques were combined with immunology that a clear picture emerged. With the aid of microelectrodes inserted into single neural end-plates in muscle it was shown that the only abnormality in the myasthenic neuromuscular junction is the size of the quanta of acetylcholine formed in the presynaptic vesicles. ${ }^{7}$ These small packages of the chemical "transmitter" are formed all the time at the rate of 1 or 2 per second and can be detected as the "micro end-plate potentials" to which they give rise in resting fibres.

When the nerve is stimulated there is a synchronous discharge of 100-200 of these quanta, and this creates enough energy to initiate an "action potential" in the postjunctional membrane, which then leads to contraction of the muscle fibres. In myasthenia gravis the quanta are only about onefifth the normal size, and despite a normal number of quanta discharged the nerve impulse fails to generate enough potential for the muscle to contract. ${ }^{8}$ All the other parts of this com- plicated sequence of events were found to be normal in myasthenic muscle.

How can this be explained in immunological terms? By immunizing animals with as little as $5 \mu \mathrm{g}$ of a thymic extract in Freund adjuvant G. Goldstein ${ }^{9}$ and his associates produced an experimental thymitis and a defect in neuromuscular transmission which appears to mimic that of myasthenia gravis. This work has now been confirmed by others. ${ }^{10}$ Microelectrical measurements suggest that the acetylcholine quanta in the end-plate potentials of these animals are abnormally small.11 Goldstein postulated that the thymus normally secretes a hormone involved in the regulation of neuromuscular transmission, which he has called "thymin." This hormone has been extracted from calf thymus, ${ }^{9}$ and experiments have shown that it can reduce the size of acetylcholine quanta in rats. ${ }^{12}$ Biochemical analysis of the neuromuscular inhibitor found in the serum of patients with myasthenia gravis ${ }^{13}$ tends to support the idea that it is a hormone present in excess. It is probably a polypeptide of small size, a feature already suggested by the temporary improvement in muscle strength seen in myasthenic patients after haemodialysis. ${ }^{14}$

The conclusion must be that in myasthenia gravis an antibody is stimulating the epithelial cells of the thymus to secrete excessive amounts of thymin, just as in thyrotoxicosis the long-acting thyroid stimulator (LATS), which is now known to be an IgG autoantibody, stimulates the thyroid cells to produce excessive amounts of thyroxine. ${ }^{15}$ In neonatal myasthenia the thymus-stimulating antibody presumably goes through the placenta and acts on the baby's own thymus, which produces too much thymin for three to six weeks, corresponding to the half life of IgG. This situation parallels neonatal thyrotoxicosis, in which LATS has been detected in the mother and baby and the disease also lasts for a few weeks only. ${ }^{16}$ Another interesting parallel between LATS and the thymus-stimulating antibody is the difficulty in suppressing them with steroids. In both instances only massive doses are temporarily effective.

The new concepts on the pathogenesis of myasthenia gravis explain the thymus lesions, the overlap with thyrotoxicosis and other autoimmune disorders, the presence of multitiple antibodies, the spontaneous exacerbations and remissions typical of all these diseases, and the good effects of 
thymectomy. The incomplete postoperative cure in longstanding or severe myasthenia is due to an associated myositis present in many cases and to the permanent loss of muscle fibres and neuromuscular junctions observed histologically. The myositic lesions are also seen in animals immunized with thymus or muscle and are explained by the cross-reactive antigens.

The most interesting problem raised by Goldstein's work is the possible physiological role of thymin in muscular activity and why total thymectomy after the neonatal period does not cause signs of deficiency in the regulation of muscle function. The main role of the thymus is its regulation of the immunity apparatus, ${ }^{17}$ especially cell-mediated immunity, and cooperation with antibody-producing cells. ${ }^{18}$ But it may have other still obscure endocrine functions to be discovered in the future.

1 Smithers, D. W., Fournal of the Faculty of Radiologists, 1959, 10, 3.

2 Simpson, J. A., Scottish Medical fournal, 1960, 5, 419.

3 Strauss, A. J. L., et al., Proceedings of the Society for Experimental Biology and Medicine, 1960, 105, 184.

- Van der Geld, H., and Oosterhuis, H. J. G. H., Vox Sanguinis,1963, 8,196.

Van der Geld, $\mathrm{H}$., and Oosterhuis, $\mathrm{H}$.

- Perlo, V. P., Neurology, 1966, 16, 431

Elmqvist, D., Hofman, W. W., Kugelberg, J., and Quastel, D. M. J., Fournal of Physiology, 1964, 174, 417 .

Thesleff, S., Annals of the New York Academy of Sciences, 1966, 135, 195. - Goldstein, G., Lancet, 1968, 2, 119.

Kalden, J. R., Williamson, W. G., Johnston, R. J., and Irvine, W. J., Clinical and Experimental Immunology, 1969, 5, 319.

1 Goldstein, G., and Hofmann, W. W., fournal of Neurology, Neurosurgery and Psychiatry, 1968, 31, 453.

12 Goldstein, G., and Hofmann, W. W., Clinical and Experimental Immunology, 1969, 4, 181.

13 Nakao, K., and Wada, F., Medical Fournal of Osaka University, 1968,

14 Stricker, E., Tholen, H., Massini, M. A., and Staub, H., fournal of Neurology, Neurosurgery and Psychiatry, 1960, 23, 291.
.

15 Adams, D. D., British Medical fournal, 1965, 1, 1015

16 McKenzie, J. M., Fournal of Clinical Endocrinology and Metabolism, 1964, 24, 660 .

17 Miller, J. F. A. P., and Mitchell, G. F., Transplantation Reviews, 1969, $1,3$.

18 Roitt, I. M., Graves, M. F., Torrigiani, G., Brostoff, J., and Playfair, J. H. L., Lancet, 1969, 2, 367.

\section{Atheroma: A New Hypothesis}

For many years pathologists have been struck by the pattern of distribution of early atheromatous lesions in the aorta. In man the lesions are typically seen close to the mouths of branch arteries. A similar distribution of lesions in the aorta also occurs in animals-for example, in rabbits fed on cholesterol. The obvious conclusion has therefore been that turbulence of blood flow at arterial junctions in some way damages the wall, thus rendering it liable to atheroma. But this general theory has certain drawbacks. It does not account for the predilection of atheroma for the abdominal rather than the thoracic aorta, in which damage to the wall from turbulence might be particularly expected.

Now C. G. Caro and his colleagues at Imperial College, ${ }^{1}$ London, have published a detailed analysis of the distribution and form of early atheromatus lesions in the human aorta and in animals given cholesterol. They draw attention to a difference in the type of lesion occurring in these two conditions. In the human aorta, the "flow divider"-that part of the artery separating the stream going to the branch artery from the main aortic stream-is commonly spared from atheroma, and cholesterol is deposited principally on the lateral part of the branch artery's orifice. But in animals with artificially raised levels of blood cholesterol the atheroma is deposited preferentially on the "flow divider" itself. Caro and his colleagues have proposed an ingenious hypothesis to account for this apparent anomaly.

Instead of invoking turbulence they attribute the distribution of lesions to alterations in the mass transport of cholesterol into and out of the arterial intima in acccrdance with the changes in shear of the flowing blood against the wall. When, as is commonly believed to happen, cholesterol is being synthesized within arterial walls and being carried away in the blood by diffusion at the intimal surface, the rate of diffusion will depend on its concentration at that surface. The faster the flow of blood and the higher the shear at the wall, the faster will cholesterol be carried away and the lower will be its concentration within the wall. Conversely, where the flow is sluggish and the shear low, the diffusion of cholesterol from wall to lumen will be slow, and therefore the concentration of cholesterol will tend to be relatively high. Such a hypothesis would explain the predilection of atheroma for the lateral part of the orifice of a branch artery and the sparing of the "flow divider."

One merit of this hypothesis is that it accounts for the relative freedom of the thoracic aorta from atheroma. This can be attributed to the faster rate of flow there than in the abdominal aorta, which is especially liable to develop lesions. Since the shear against the wall rises peripherally in the arterial system, ${ }^{2}$ and is also high in capillaries, ${ }^{3}$ the hypothesis would also account for the relative freedom of small vessels from atheroma. The hypothesis is perhaps compatible with the idea that exercise staves off atheroma, because an increased rate of blood flow would tend to lower the concentration of cholesterol in the arterial wall. In experimental animals fed cholesterol the cholesterol is deposited, in contrast, by diffusion from the blood, where its concentration is high, to the intima, where its concentration is lower. Thus the highest gradient for diffusion would occur in those regions where flow was faster and shear greater, notably the "flow dividers."

This interesting work seems to meet the first requirement of any hypothesis, which is to explain all the known facts. Another interpretation of the observations in man, involving the tendency of platelets to aggregate particularly at areas of relatively low flow, could be made in terms of the J. B. Duguid's hypothesis, ${ }^{4}$ in which local thrombus formed on the arterial intima is the precursor of an atheromatous plaque. However, this does not account for the type of lesion found in the cholesterol feeding of animals. Problems which remain are the association of atheroma with hypertension, and the sparing of veins.

It is interesting to speculate on the teleological consequences of the hypothesis, for the implication of it seems to be that large arteries contain within them the seeds of their own destruction by secreting cholesterol. The harm can be checked only if wall shear is kept high. Why should arterial walls be producing cholesterol if the material simply diffuses out into the lumen? It is tempting to suppose that the cholesterol or a derivative of it is concerned with that property unique to the walls of blood vessels-inhibition of clot formation on their surface. Though the new hypothesis goes only part of the way towards explaining the genesis of atheroma, in such a difficult field we must be grateful for any light that can be shed in dark places.

\footnotetext{
1 Caro, C. G., Fitz-Gerald, J. M., Schroter, R. C., Proceedings of the Royal Society, Series B, 1971, 177, 109

Whitmore, R. L., Nature, 1967, 215, 123.

3 Fitz-Gerald, J. M., Proceedings of the Royal Society, Series B, 1969, 174, 193.

- Duguid, J. B., Fournal of Pathology and Bacteriology, 1946, 58, 207.
} 